\title{
An Offline Formulation of MPC for LPV Systems Using Linear Matrix Inequalities
}

\author{
P. Bumroongsri \\ Department of Chemical Engineering, Faculty of Engineering, Mahidol University, Phuttamonthon 4 Road, \\ Salaya, Nakhon Pathom 73170, Thailand
}

Correspondence should be addressed to P. Bumroongsri; pornchai.bum@mahidol.ac.th

Received 15 January 2014; Accepted 21 March 2014; Published 22 April 2014

Academic Editor: Jong Hae Kim

Copyright (C) 2014 P. Bumroongsri. This is an open access article distributed under the Creative Commons Attribution License, which permits unrestricted use, distribution, and reproduction in any medium, provided the original work is properly cited.

An offline model predictive control (MPC) algorithm for linear parameter varying (LPV) systems is presented. The main contribution is to develop an offline MPC algorithm for LPV systems that can deal with both time-varying scheduling parameter and persistent disturbance. The norm-bounding technique is used to derive an offline MPC algorithm based on the parameterdependent state feedback control law and the parameter-dependent Lyapunov functions. The online computational time is reduced by solving offline the linear matrix inequality (LMI) optimization problems to find the sequences of explicit state feedback control laws. At each sampling instant, a parameter-dependent state feedback control law is computed by linear interpolation between the precomputed state feedback control laws. The algorithm is illustrated with two examples. The results show that robust stability can be ensured in the presence of both time-varying scheduling parameter and persistent disturbance.

\section{Introduction}

Model predictive control (MPC), also known as receding horizon control, is an effective multivariable control algorithm in which a dynamic optimization problem is solved online. At each sampling time, MPC solves a finite horizon optimal control problem based on an explicit model of the plant. Although an optimal control sequence is determined, only the first control action is applied to the plant. Due to its ability to guarantee optimality while ensuring the satisfaction of constraints on input and state, MPC has received much interest in both industry and academia [1-3].

An explicit linear model is typically used in the MPC formulation because the online optimization can be reduced to either a linear program or a quadratic program. Since an industrial process is inherently nonlinear to a certain extent, the control performance of linear MPC can deteriorate as operating conditions significantly change $[4,5]$. For this reason, MPC for linear parameter varying (LPV) systems has been widely developed. LPV systems are linear systems whose dynamics depend on the scheduling parameter that can be measured online. The analysis and synthesis of LPV systems play an important role in control theory since nonlinear systems can be dealt within the framework of LPV systems $[6,7]$.

In the context of MPC for LPV systems, one of the main approaches is to solve a semidefinite problem under linear matrix inequality (LMI) [8]. Quasi-Min-Max MPC algorithm for LPV systems was developed by $\mathrm{Lu}$ and Arkun [9]. Although the scheduling parameter is included in the controller design, it is assumed that there is no disturbance present in the problem formulation, so the algorithm cannot deal with disturbance. MPC for LPV systems using parameter-dependent Lyapunov functions was developed by [10]. It is shown that the proposed MPC algorithm can achieve less conservative results as compared with a robust MPC algorithm derived by using a single Lyapunov function [4]. However, this algorithm includes only time-varying scheduling parameter in the problem formulation so it cannot ensure robust stability in the presence of disturbance. The 
bound on the rate of variation of the scheduling parameter can also be taken into account in the MPC formulation $[11,12]$. However, this technique is not applicable to the case where there is the disturbance acting on the system.

MPC for LPV systems can be designed by using ellipsoidal set prediction $[13,14]$. At each sampling instant, the predicted future states on the finite horizon are bounded by using a sequence of ellipsoids. The terminal ellipsoid is contained in a target set guaranteeing stability. The main drawback of this approach lies in the fact that the computational load increases with the length of prediction horizon. In order to reduce online computational time, an offline MPC algorithm for LPV systems was developed [15]. The realtime state feedback gain is calculated by linear interpolation between the precomputed state feedback gains. Although the online computational time is significantly reduced, the disturbance is not taken into account in the offline MPC formulation so robust stability cannot be guaranteed in the presence of disturbance. Explicit MPC for LPV systems was proposed by Besselmann et al. [16]. Only time-varying scheduling parameter is included in the problem formulation and it is also assumed that there is no disturbance. In Ding [17, 18], both time-varying scheduling parameter and disturbance are included in the problem formulation. However, the optimization problem contains a lot of decision variables and constraints, so the algorithm is computationally prohibitive in practical situations.

In the context of tube-based MPC [19-21], the disturbance is explicitly taken into account in the MPC design. The basic concept of robust tube-based MPC is to compute the region around the nominal prediction that contains any possible states of the uncertain system. One of the main advantages is that its online computational complexity increases only linearly with the prediction horizon. However, the timevarying scheduling parameter is not incorporated into the MPC design, so robust stability cannot be ensured in the presence of parametric uncertainty.

In this paper, an offline MPC algorithm for LPV systems is presented. Unlike Wan and Kothare [22] where only timevarying scheduling parameter is considered in the offline MPC formulation, the main contribution of this paper is to develop an offline MPC algorithm for LPV systems that can deal with both persistent disturbance and time-varying scheduling parameter. The norm-bounding technique [23] is used to derive an offline MPC algorithm based on the parameter-dependent state feedback control law and the parameter-dependent Lyapunov functions. Most of the optimization problems are solved offline, so the developed MPC algorithm can be applied to fast processes. This article is organized as follows. Section 2 concerns with problem statement and control objectives. The proposed algorithm is described in Section 3. In Section 4, the effectiveness of the proposed MPC algorithm is illustrated. Finally, Section 5 presents some conclusions.

Notation. For any vector $x$ and positive-definite matrix $P$, $\|x\|_{P}^{2}=x^{T} P x . x(k)$ is the state measured at real-time $k$ and $x(k+i \mid k)$ is the state at prediction time $k+i$ predicted at real-time $k$. The symbol $*$ denotes symmetric blocks in matrices. An element belonging to a convex hull $\mathrm{Co}\{\cdot\}$ means that it is a convex combination of the elements in $\{\cdot\}$. The time-dependence $(k)$ of the MPC decision variables is often dropped for simplicity. $I$ is the identity matrix with appropriate dimension.

\section{Problem Statement}

Consider the following discrete-time LPV system:

$$
\begin{gathered}
x(k+1)=A(p(k)) x(k)+B u(k)+D(\alpha(k)) v(k), \\
y(k)=C(p(k)) x(k),
\end{gathered}
$$

where $x(k) \in \mathfrak{R}^{n_{x}}$ denotes the state, $u(k) \in \mathfrak{R}^{n_{u}}$ denotes the control input, $v(k) \in \Re^{n_{v}}$ denotes the disturbance, and $y(k) \in \mathfrak{R}^{n_{y}}$ denotes the output. The superscripts $n_{x}, n_{u}$, $n_{v}$, and $n_{y}$ denote the dimensions of $x(k), u(k), v(k)$, and $y(k)$, respectively. It is assumed that the time-varying scheduling parameter $p(k)=\left[p_{1}(k), p_{2}(k), \ldots, p_{L}(k)\right] \in \mathfrak{R}^{L}$ is measurable at each sampling time but its future values are considered to be uncertain. The input and output constraints are

$$
\begin{array}{ll}
|u(k)| \leq \bar{u}, \quad \bar{u}_{h}>0, \quad h \in\left\{1,2, \ldots, n_{u}\right\}, \\
|y(k)| \leq \bar{y}, \quad \bar{y}_{r}>0, \quad r \in\left\{1,2, \ldots, n_{y}\right\} .
\end{array}
$$

Any $A(p(k))$ and $C(p(k))$ belong to a convex polytope $\Omega$ defined by

$$
\Omega=\operatorname{Co}\left\{\left[A_{1}, C_{1}\right],\left[A_{2}, C_{2}\right], \ldots,\left[A_{L}, C_{L}\right]\right\},
$$

so they can be written as

$$
\begin{gathered}
{[A(p(k)), C(p(k))]=\sum_{j=1}^{L} p_{j}(k)\left[A_{j}, C_{j}\right],} \\
\sum_{j=1}^{L} p_{j}(k)=1, \quad 0 \leq p_{j}(k) \leq 1,
\end{gathered}
$$

where $\left[A_{j}, C_{j}\right]$ are the vertices of $\Omega$ and $L$ is the number of the vertices of $\Omega$. Any $D(\alpha(k))$ belongs to a convex polytope $\Omega_{D}$ defined by

$$
\Omega_{D}=\operatorname{Co}\left\{D_{1}, D_{2}, \ldots, D_{n_{D}}\right\}
$$

so it can be written as

$$
\begin{gathered}
D(\alpha(k))=\sum_{t=1}^{n_{D}} \alpha_{t}(k) D_{t}, \\
\sum_{t=1}^{n_{D}} \alpha_{t}(k)=1, \quad 0 \leq \alpha_{t}(k) \leq 1,
\end{gathered}
$$


where $D_{t}$ are the vertices of $\Omega_{D}, n_{D}$ is the number of the vertices of $\Omega_{D}$, and $\alpha_{t}(k)$ is the time-varying parameter that is not necessary to be measurable. The disturbance $v(k)$ is unmeasurable and persistent. It is assumed to lie in a convex polytope $\Omega_{v}$ defined by

$$
\Omega_{v}=\operatorname{Co}\left\{v_{1}, v_{2}, \ldots, v_{m_{v}}\right\}
$$

where $m_{v}$ is the number of the vertices of $\Omega_{v}$. The objective is to find a parameter-dependent state feedback control law $K(p(k+i))=\sum_{j=1}^{L} p_{j}(k+i) K_{j}$, where $K_{j}, j \in\{1,2, \ldots, L\}$ are the state feedback gains corresponding to the vertices of $\Omega$ that is able to guarantee both robust stability and constraint satisfaction within a positively invariant set.

Definition 1. The set $Z$ is said to be positively invariant set if it has the property that whenever the current state is contained in this set $x(k) \in Z$, all possible predicted states must be contained in this set $x(k+i \mid k) \in Z$ for all admissible realizations of $p(k+i), \alpha(k+i)$ and $v(k+i), i \geq 0$.

Remark 2. In this paper, the positively invariant set is $Z=$ $\left\{x(k+i \mid k) \in \mathfrak{R}^{n_{x}} \mid\|x(k+i \mid k)\|_{P(p(k+i))}^{2} \leq \gamma\right\}$, where $P(p(k+i))=\sum_{j=1}^{L} p_{j}(k+i) P_{j}$ is a parameter-dependent Lyapunov matrix, $P_{j}, j \in\{1,2, \ldots, L\}$ are the Lyapunov matrices corresponding to the vertices of $\Omega$, and $\gamma$ is an upper bound on the infinite horizon cost. From the convexity of the polytopic description, $Z$ is an intersection area of $\varepsilon_{j}=$ $\left\{x(k+i \mid k) \in \mathfrak{R}^{n_{x}} \mid\|x(k+i \mid k)\|_{P_{j}}^{2} \leq \gamma\right\}$.

Considering the discrete-time LPV system (1) to (9) at each sampling time $k$, a parameter-dependent state feedback control law $u(k+i \mid k)=K(p(k+i)) x(k+i \mid k)$ that (i) minimizes an upper bound $\gamma$ on $J_{\infty}(k)$ and (ii) guarantees both robust stability and robust constraint satisfaction within a positively invariant set $Z$ can be calculated by solving the following optimization problem:

$$
\begin{aligned}
& \min _{\gamma, K(p(k+i)), P(p(k+i)), i \geq 0} \max _{A(p(k+i)), C(p(k+i))] \in \Omega,} \\
& D(\alpha(k+i)) \in \Omega_{D}, v(k+i) \in \Omega_{v} \\
& J_{\infty}(k)=\sum_{i=0}^{\infty}\left[\left\|x_{n}(k+i \mid k)\right\|_{\psi}^{2}+\left\|K(p(k+i)) x_{n}(k+i \mid k)\right\|_{\sigma}^{2}\right]
\end{aligned}
$$

s.t.

$$
\begin{gathered}
\left\|x_{n}(k+i+1 \mid k)\right\|_{P(p(k+i+1))}^{2}-\left\|x_{n}(k+i \mid k)\right\|_{P(p(k+i))}^{2} \\
\leq-\left[\left\|x_{n}(k+i \mid k)\right\|_{\psi}^{2}+\left\|K(p(k+i)) x_{n}(k+i \mid k)\right\|_{\sigma}^{2}\right] \\
\|x(k)\|_{P(p(k))}^{2} \leq \gamma, \quad x(k)=x_{n}(k \mid k)=x(k \mid k)
\end{gathered}
$$

$$
\begin{gathered}
\|x(k+i+1 \mid k)\|_{P(p(k+i+1))}^{2} \leq \gamma \\
|K(p(k+i)) x(k+i \mid k)| \leq \bar{u}, \\
\bar{u}_{h}>0, \quad h \in\left\{1,2, \ldots, n_{u}\right\} \\
|C(p(k+i+1)) x(k+i+1 \mid k)| \leq \bar{y}, \\
\bar{y}_{r}>0, \quad r \in\left\{1,2, \ldots, n_{y}\right\},
\end{gathered}
$$

where $x_{n}(k+i+1 \mid k)=[A(p(k+i))+B K(p(k+i))] x_{n}(k+$ $i \mid k)$ is the predicted state not corrupted by disturbance, $x(k+i+1 \mid k)=[A(p(k+i))+B K(p(k+i))] x(k+i \mid$ $k)+D(\alpha(k+i)) v(k+i)$ is the predicted state with disturbance, and $\psi$ and $\sigma$ are symmetric weighting matrices. The cost monotonicity is guaranteed by (11). A positively invariant set containing the state $x(k)$ at each sampling time is computed by (12). All predicted states $x(k+i+1 \mid k)$ are restricted to lie in a positively invariant set by (13). The input and output constraints are guaranteed by (14) and (15), respectively.

\section{Offline MPC for LPV Systems with Persistent Disturbances}

First of all, we will begin with the preliminary results of Wada et al. [10] where only time-varying scheduling parameter is considered in the problem formulation. Then, the proposed algorithm that can deal with both time-varying scheduling parameter and persistent disturbance will be developed. By following Wada et al. [10], (11) and (12) are satisfied and the cost monotonicity is guaranteed if there exist matrices $Y_{j}, G_{j}$, symmetric matrices $Q_{j}$ and a positive scalar $\gamma$ such that the following LMIs are satisfied:

$$
\left[\begin{array}{cccc}
G_{j}+G_{j}^{T}-Q_{j} & * & * & * \\
A_{j} G_{j}+B Y_{j} & Q_{l} & * & * \\
\psi^{1 / 2} G_{j} & 0 & \gamma I & * \\
\sigma^{1 / 2} Y_{j} & 0 & 0 & \gamma I
\end{array}\right] \geq 0, \quad j, l \in\{1,2, \ldots, L\}
$$

$$
\left[\begin{array}{cc}
1 & * \\
x(k) & Q_{j}
\end{array}\right] \geq 0, \quad j \in\{1,2, \ldots, L\}
$$

Then, it follows that $\gamma$ is the upper bound on $J_{\infty}(k)$. Moreover, a parameter-dependent state feedback gain is given by $K(p(k+i))=\sum_{j=1}^{L} p_{j}(k+i) K_{j}, K_{j}=Y_{j} G_{j}^{-1}$.

Next, we will present the results of this paper that can deal with both time-varying scheduling parameter and disturbance.

Proposition 3 (Robust stability in the presence of both time-varying scheduling parameter and disturbance). (13) is 
satisfied if there exist matrices $Y_{j}, G_{j}$ and symmetric matrices $Q_{j}$ such that the following LMIs are satisfied:

$$
\begin{gathered}
{\left[\begin{array}{cc}
\theta\left(G_{j}+G_{j}^{T}-Q_{j}\right) & * \\
A_{j} G_{j}+B Y_{j} & Q_{l}
\end{array}\right] \geq 0, \quad j, l \in\{1,2, \ldots, L\},} \\
{\left[\begin{array}{cc}
\xi & * \\
D_{t} v_{s} & Q_{l}
\end{array}\right] \geq 0, \quad t \in\left\{1,2, \ldots, n_{D}\right\}, s \in\left\{1,2, \ldots, m_{v}\right\}} \\
l \in\{1,2, \ldots, L\}, \quad \xi=\left(1-\theta^{1 / 2}\right)^{2},
\end{gathered}
$$

where $0<\theta<1$ is a prespecified scalar. Then, all predicted states are restricted to lie in a positively invariant set which is an intersection area of $\varepsilon_{j}=\left\{x(k+i \mid k) \in \mathfrak{R}^{n_{x}} \mid\right.$ $\left.\|x(k+i \mid k)\|_{\mathrm{Q}_{j}^{-1}}^{2} \leq 1\right\}$.

Proof. Equation (13) is guaranteed by (18) and (19). The proof detail can be found in Appendix A.

Remark 4. $\theta$ is a parameter that bounds disturbance-free state trajectories. The value of $\theta$ should be chosen such that $\theta \rightarrow 1$ as $v_{s} \rightarrow 0$.

Proposition 5 (Robust constraint satisfaction in the presence of both time-varying scheduling parameter and disturbance). Robust constraint satisfaction is guaranteed if there exist matrices $Y_{j}, G_{j}$ and symmetric matrices $Q_{j}$ such that the following LMIs are satisfied:

$$
\begin{gathered}
{\left[\begin{array}{cc}
\chi & * \\
Y_{j}^{T} & G_{j}+G_{j}^{T}-Q_{j}
\end{array}\right] \geq 0,} \\
\chi_{h h} \leq \bar{u}_{h}^{2}, \quad h \in\left\{1,2, \ldots, n_{u}\right\}, \quad j \in\{1,2, \ldots, L\}, \\
\quad\left[\begin{array}{c}
\Gamma \\
\left(A_{j} G_{j}+B Y_{j}\right)^{T} C_{l}^{T} G_{j}+G_{j}^{T}-Q_{j}
\end{array}\right] \geq 0, \\
\Gamma_{r r} \leq \Xi_{r}, \\
\Xi_{r}=\left(\bar{y}_{r}-\Phi_{r}^{1 / 2}\right)^{2}, \quad r \in\left\{1,2, \ldots, n_{y}\right\}, \quad j, l \in\{1,2, \ldots, L\},
\end{gathered}
$$

where $\Phi_{r}$ is a scalar that can be obtained by solving the following optimization problem

$$
\begin{array}{ll}
\min _{\Phi_{r}} & \Phi_{r} \\
\text { s.t. } & {\left[\begin{array}{cc}
\Phi_{r} & * \\
C_{r, l} D_{t} v_{s} & 1
\end{array}\right] \geq 0,} \\
& r \in\left\{1,2, \ldots, n_{y}\right\}, l \in\{1,2, \ldots, L\}, \\
& t \in\left\{1,2, \ldots, n_{D}\right\}, s \in\left\{1,2, \ldots, m_{v}\right\},
\end{array}
$$

where $C_{r, l}$ is the rth row of $C_{l}$.

Proof. The input constraint (14) is guaranteed by (20). The proof details can be found in Appendix B. The output constraint (15) is guaranteed by (21). The proof details can be found in Appendix C.

By considering Propositions 3 and 5, a parameter-dependent state feedback control law that guarantees both robust stability and robust constraint satisfaction can be calculated. Consider the discrete-time LPV system (1) to (9) at each sampling instant $k$, a parameter-dependent state feedback control law $u(k+i \mid k)=K(p(k+i)) x(k+i \mid k), K(p(k+$ $i))=\sum_{j=1}^{L} p_{j}(k+i) K_{j}, K_{j}=Y_{j} G_{j}^{-1}$ that guarantees both robust stability and robust constraint satisfaction within a positively invariant set $Z$ is obtained by solving the following optimization problem:

$$
\min _{\gamma, Y_{j}, G_{j}, Q_{j}} \gamma
$$

$$
\text { s.t. } \quad(16) \text { to }(23) \text {. }
$$

It is computationally demanding to solve the optimization problem (24) at each sampling time. Inspired by Bumroongsri and Kheawhom [15], we propose an offline MPC algorithm for LPV systems that transfer most of the computations offline.

\section{Algorithm 6.}

Offline. Choose a sequence of states $x_{i}, i \in\{1,2, \ldots, N\}$. For each $i$, substitute $x(k)$ in (17) by $x_{i}$ and solve the optimization problem (24) to obtain the corresponding feedback gains $K_{i, j}=Y_{i, j} G_{i, j}^{-1}$ and ellipsoids $\varepsilon_{i, j}=\left\{x \in \Re^{n_{x}} \mid x^{T} Q_{i, j}^{-1} x \leq 1\right\}$, where the subscript $i, j$ denote the solution of (24) for each $x_{i}, i \in\{1,2, \ldots, N\}$ and vertices $j, j \in\{1,2, \ldots, L\}$. Note that (i) the positively invariant set for each $i$ is an intersection area of $\varepsilon_{i, j}, j \in\{1,2, \ldots, L\}$ (ii) the inequalities (26) should be satisfied for each $i \neq N$ to ensure robust stability of the offline algorithm

$$
\theta Q_{i, j}^{-1}-\left(A_{j}+B K_{i+1, j}\right)^{T} Q_{i, l}^{-1}\left(A_{j}+B K_{i+1, j}\right) \geq 0 .
$$

Remark 7. A sequence of states $x_{i}, i \in\{1,2, \ldots, N\}$ should be chosen such that for each $j$, the ellipsoids $\varepsilon_{i, j}, \forall i \neq N$ obtained are nested $\left(\varepsilon_{i+1, j} \subset \varepsilon_{i, j}, Q_{i+1, j}<Q_{i, j}\right)$. This is to guarantee that the state is kept within $\varepsilon_{i, j}$ and driven towards $\varepsilon_{i+1, j}$, and so on.

Online. At each sampling time $k$, measure $p(k)$ and $x(k)$. If $x(k) \in \varepsilon_{i, j}, x(k) \notin \varepsilon_{i+1, j}, j \in\{1,2, \ldots, L\}, i \neq N$, adopt the following control law:

$$
\begin{gathered}
u(k)=K(\lambda(k)) x(k), \\
K(\lambda(k))=\lambda(k)\left[\sum_{j=1}^{L} p_{j}(k) K_{i, j}\right] \\
+(1-\lambda(k))\left[\sum_{j=1}^{L} p_{j}(k) K_{i+1, j}\right],
\end{gathered}
$$


where $\lambda(k) \in(0,1]$ is calculated by solving

$$
\begin{aligned}
x(k)^{T}\left(\lambda(k)\left[\sum_{j=1}^{L} p_{j}(k) Q_{i, j}^{-1}\right]\right. \\
\left.+(1-\lambda(k))\left[\sum_{j=1}^{L} p_{j}(k) Q_{i+1, j}^{-1}\right]\right) x(k)=1 .
\end{aligned}
$$

If $x(k) \in \varepsilon_{N, j}, j \in\{1,2, \ldots, L\}$, adopt the following control law:

$$
u(k)=K_{N} x(k), \quad K_{N}=\sum_{j=1}^{L} p_{j}(k) K_{N, j} .
$$

An intersection area of $\varepsilon_{1, j}, j \in\{1,2, \ldots, L\}$ is the largest positively invariant set where robust stability and robust constraint satisfaction are guaranteed. If $x(k) \in \varepsilon_{1, j}, j \in$ $\{1,2, \ldots, L\}$, then $x(k+i) \in \varepsilon_{1, j}, j \in\{1,2, \ldots, L\}, i \in$ $\{0,1, \ldots, \infty\}$. Thus, Algorithm 6 is always feasible if it is initially feasible.

Remark 8. The proposed offline MPC algorithm can deal with both time-varying scheduling parameter and disturbance. In comparison, an offline MPC algorithm [22] cannot deal with disturbance.

\section{Examples}

The numerical simulations have been performed in Intel Core 2 Duo (2.53 GHz), 2 GB RAM, using SeDuMi [24] and YALMIP [25] within the Matlab R2008a environment.

Example 1. The first example is a continuous stirred tank reactor (CSTR) adapted from Ding and Huang [26] where an exothermic reaction $A \rightarrow B$ takes place. The dynamic model based on a component balance and an energy balance can be written as

$$
\begin{gathered}
\dot{C}_{A}=\frac{q}{V}\left(C_{A F}-C_{A}\right)-k_{o} e^{(-E a / R T)} C_{A}+D_{C} v, \\
\dot{T}=\frac{q}{V}\left(T_{f}-T\right)+\frac{-\Delta H}{\rho C_{p}} k_{o} e^{(-E a / R T)} C_{A} \\
+\frac{U A}{V \rho C_{p}}\left(T_{c}-T\right)+D_{T} v,
\end{gathered}
$$

where $C_{A}$ denotes the concentration of $A$ in the reactor, $T$ denotes the reactor temperature, $T_{c}$ denotes the temperature of the coolant stream, and $v$ denotes the disturbance acting on the system. The operating parameters are shown in Table 1.

By defining $\bar{C}_{A}=C_{A}-C_{A \text {,eq }}, \bar{T}=T-T_{\text {eq }}$ and $\bar{T}_{c}=$ $T_{c}-T_{c, \text { eq }}$, (31) and (32) can be transformed into the fllowing nonlinear model:

$$
\left[\begin{array}{c}
\dot{\bar{C}}_{A} \\
\hline \bar{T}
\end{array}\right]=\left\{\begin{array}{c}
{\left[\begin{array}{cc}
-\frac{q}{V}-\varphi_{1}(\bar{T}) & -\varphi_{2}(\bar{T}) \\
\left(\frac{-\Delta H}{\rho C_{p}}\right) \varphi_{1}(\bar{T})-\frac{q}{V}-\frac{U A}{V \rho C_{p}}+\left(\frac{-\Delta H}{\rho C_{p}}\right) \varphi_{2}(\bar{T})
\end{array}\right]\left[\begin{array}{c}
\bar{C}_{A} \\
\bar{T}
\end{array}\right]+\left[\begin{array}{c}
0 \\
\frac{U A}{V \rho C_{p}}
\end{array}\right] \bar{T}_{c}+\left[\begin{array}{l}
D_{C} \\
D_{T}
\end{array}\right] v, \bar{T} \neq 0} \\
{\left[\begin{array}{cc}
-\frac{q}{V}-\varphi_{1}(\bar{T}) & 0 \\
\left(\frac{-\Delta H}{\rho C_{p}}\right) \varphi_{1}(\bar{T}) & -\frac{q}{V}-\frac{U A}{V \rho C_{p}}
\end{array}\right]\left[\begin{array}{c}
\bar{C}_{A} \\
\bar{T}
\end{array}\right]+\left[\begin{array}{c}
0 \\
\frac{U A}{V \rho C_{p}}
\end{array}\right] \bar{T}_{c}+\left[\begin{array}{c}
D_{C} \\
D_{T}
\end{array}\right] v, \bar{T}=0}
\end{array}\right\},
$$

where $\varphi_{1}(\bar{T})=k_{o} \exp \left(-\left(E_{a} / R\right) /\left(\bar{T}+T_{\mathrm{eq}}\right)\right), \varphi_{2}(\bar{T})=$ $k_{o}\left[\exp \left(-\left(E_{a} / R\right) /\left(\bar{T}+T_{\mathrm{eq}}\right)\right)-\exp \left(-\left(E_{a} / R\right) / T_{\mathrm{eq}}\right)\right] C_{A, \mathrm{eq}} / \bar{T}$. For
$|\bar{T}| \leq \beta$, (33) is transformed into the following model in order to balance the submodels:

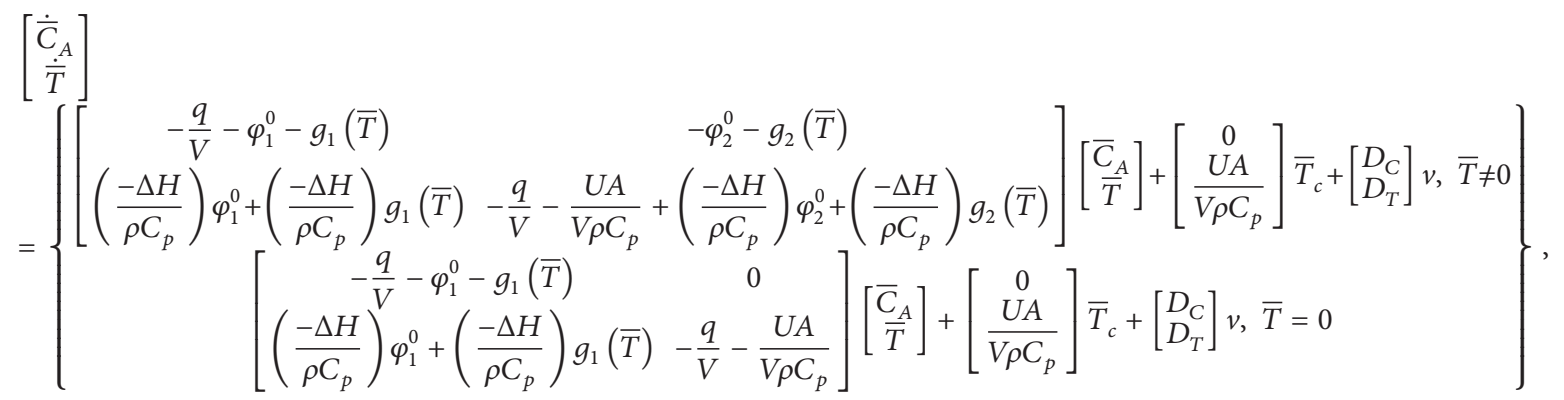

where $g_{1}(\bar{T})=\varphi_{1}(\bar{T})-\varphi_{1}^{0}, g_{2}(\bar{T})=\varphi_{2}(\bar{T})-\varphi_{2}^{0}, \varphi_{1}^{0}=\left[\varphi_{1}(-\beta)+\right.$ $\left.\varphi_{1}(\beta)\right] / 2$ and $\varphi_{2}^{0}=\left[\varphi_{2}(-\beta)+\varphi_{2}(\beta)\right] / 2$. Since $g_{1}(\bar{T})$ and $g_{2}(\bar{T})$ can vary between $g_{1}(-\beta) \leq g_{1}(\bar{T}) \leq g_{1}(\beta)$ and $g_{2}(-\beta) \leq$ $g_{2}(\bar{T}) \leq g_{2}(\beta)$, we have that all the solutions of (34) are also the solutions of the following differential inclusion:

$$
\left[\begin{array}{c}
\dot{\bar{C}}_{A} \\
\dot{\bar{T}}
\end{array}\right] \in\left(\sum_{j=1}^{4} p_{j} A_{j}\right)\left[\begin{array}{c}
\bar{C}_{A} \\
\bar{T}
\end{array}\right]+\left[\begin{array}{c}
0 \\
\frac{U A}{V \rho C_{p}}
\end{array}\right] \bar{T}_{c}+\left[\begin{array}{c}
D_{C} \\
D_{T}
\end{array}\right] v
$$


where $A_{j}$ are given by

$$
\begin{aligned}
& A_{1} \\
& =\left[\left(\frac{-\Delta H}{\rho C_{p}}\right) \varphi_{1}^{0}+2\left(\frac{-\Delta H}{\rho C_{p}}\right) g_{1}(\beta)-\frac{q}{V}-\frac{U A}{V \rho C_{p}}+\left(\frac{-\Delta H}{\rho C_{p}}\right) \varphi_{2}^{0}\right], \\
& A_{2}^{0}-2 g_{1}(\beta) \\
& =\left[\left(\frac{-\Delta H}{\rho C_{p}}\right)^{0} \varphi_{1}^{0}+2\left(\frac{-\Delta H}{\rho C_{p}}\right) g_{1}(-\beta)-\frac{q}{V}-\frac{U A}{V \rho C_{p}}+\left(\frac{-\Delta H}{\rho C_{p}}\right) \varphi_{2}^{0}\right],
\end{aligned}
$$

$A_{3}$

$$
=\left[\begin{array}{cc}
-\frac{q}{V}-\varphi_{1}^{0} & -\varphi_{2}^{0}-2 g_{2}(\beta) \\
\left(\frac{-\Delta H}{\rho C_{p}}\right) \varphi_{1}^{0} & -\frac{q}{V}-\frac{U A}{V \rho C_{p}}+\left(\frac{-\Delta H}{\rho C_{p}}\right) \varphi_{2}^{0}+2\left(\frac{-\Delta H}{\rho C_{p}}\right) g_{2}(\beta)
\end{array}\right],
$$

$$
\begin{aligned}
& A_{4} \\
& =\left[\begin{array}{cc}
-\frac{q}{V}-\varphi_{1}^{0} & -\varphi_{2}^{0}-2 g_{2}(-\beta) \\
\left(\frac{-\Delta H}{\rho C_{p}}\right) \varphi_{1}^{0} & -\frac{q}{V}-\frac{U A}{V \rho C_{p}}+\left(\frac{-\Delta H}{\rho C_{p}}\right) \varphi_{2}^{0}+2\left(\frac{-\Delta H}{\rho C_{p}}\right) g_{2}(-\beta)
\end{array}\right]
\end{aligned}
$$

and $p_{j}$ are given by

$$
\begin{aligned}
& p_{1}=\frac{1}{2} \frac{\left(g_{1}(\bar{T})-g_{1}(-\beta)\right)}{\left(g_{1}(\beta)-g_{1}(-\beta)\right)}, \\
& p_{2}=\frac{1}{2} \frac{\left(g_{1}(\beta)-g_{1}(\bar{T})\right)}{\left(g_{1}(\beta)-g_{1}(-\beta)\right)}, \\
& p_{3}=\frac{1}{2} \frac{\left(g_{2}(\bar{T})-g_{2}(-\beta)\right)}{\left(g_{2}(\beta)-g_{2}(-\beta)\right)}, \\
& p_{4}=\frac{1}{2} \frac{\left(g_{2}(\beta)-g_{2}(\bar{T})\right)}{\left(g_{2}(\beta)-g_{2}(-\beta)\right)} .
\end{aligned}
$$

The objective is to regulate $\bar{C}_{A}$ and $\bar{T}$ by manipulating $\bar{T}_{c}$. The input and output constraints are $\left|\bar{C}_{A}\right| \leq 0.5 \mathrm{~mol} / \mathrm{L},|\bar{T}| \leq 5 \mathrm{~K}$ and $\left|\bar{T}_{c}\right| \leq 40 \mathrm{~K}$. The discrete-time LPV model is obtained by discretizing (35) with a sampling period of $0.01 \mathrm{~min}$ and it is omitted here for brevity. The symmetric weighting matrices in (10) are $\Psi=\left[\begin{array}{ll}1 & 0 \\ 0 & 0\end{array}\right]$ and $\sigma=0.1$. The value of $\theta$ in (18) has been fixed at 0.97 which has negligible influence on the control performance.

Figure 1(a) shows the ellipsoids computed offline by Algorithm 6. In this example, four sequences of ellipsoids are computed because the polytope $\Omega$ has four vertices. A sequence of positively invariant sets computed by intersection among four sequences of ellipsoids is shown in Figure 1(b).

Figure 2 shows the closed-loop responses of the system when the disturbances are varied as $v(t)=0.1 \sin (10 t)$, $0.05 \sin (10 t)$, and $0.001 \sin (10 t)$, respectively. It can be observed that $\bar{C}_{A}$ and $\bar{T}$ are bounded for all values of disturbances so robust stability is ensured by applying Algorithm 6 .

The online computational time of Algorithm 6 is very low as shown in Table 2 so it is applicable to fast systems.

Example 2. The second example is an angular positioning system adapted from Kothare et al. [4]. The system consists of an electric motor driving a rotating antenna so that is always points in the direction of a moving object. The motion of the antenna can be described by the following discrete-time LPV model:

$$
\begin{aligned}
& {\left[\begin{array}{l}
x_{1}(k+1) \\
x_{2}(k+1)
\end{array}\right]=} {\left[\begin{array}{cc}
1 & 0.1 \\
0 & 1-0.1 \Delta(k)
\end{array}\right]\left[\begin{array}{l}
x_{1}(k) \\
x_{2}(k)
\end{array}\right] } \\
&+\left[\begin{array}{c}
0 \\
0.0787
\end{array}\right] u(k)+\left[\begin{array}{l}
0.15 \\
0.15
\end{array}\right] v(k), \\
& y(k)=\left[\begin{array}{ll}
1 & 0 \\
0 & 1
\end{array}\right]\left[\begin{array}{l}
x_{1}(k) \\
x_{2}(k)
\end{array}\right]
\end{aligned}
$$

where $x_{1}(k)$ is the angular position of the antenna, $x_{2}(k)$ is the angular velocity of the antenna, $u(k)$ is the input voltage to the 

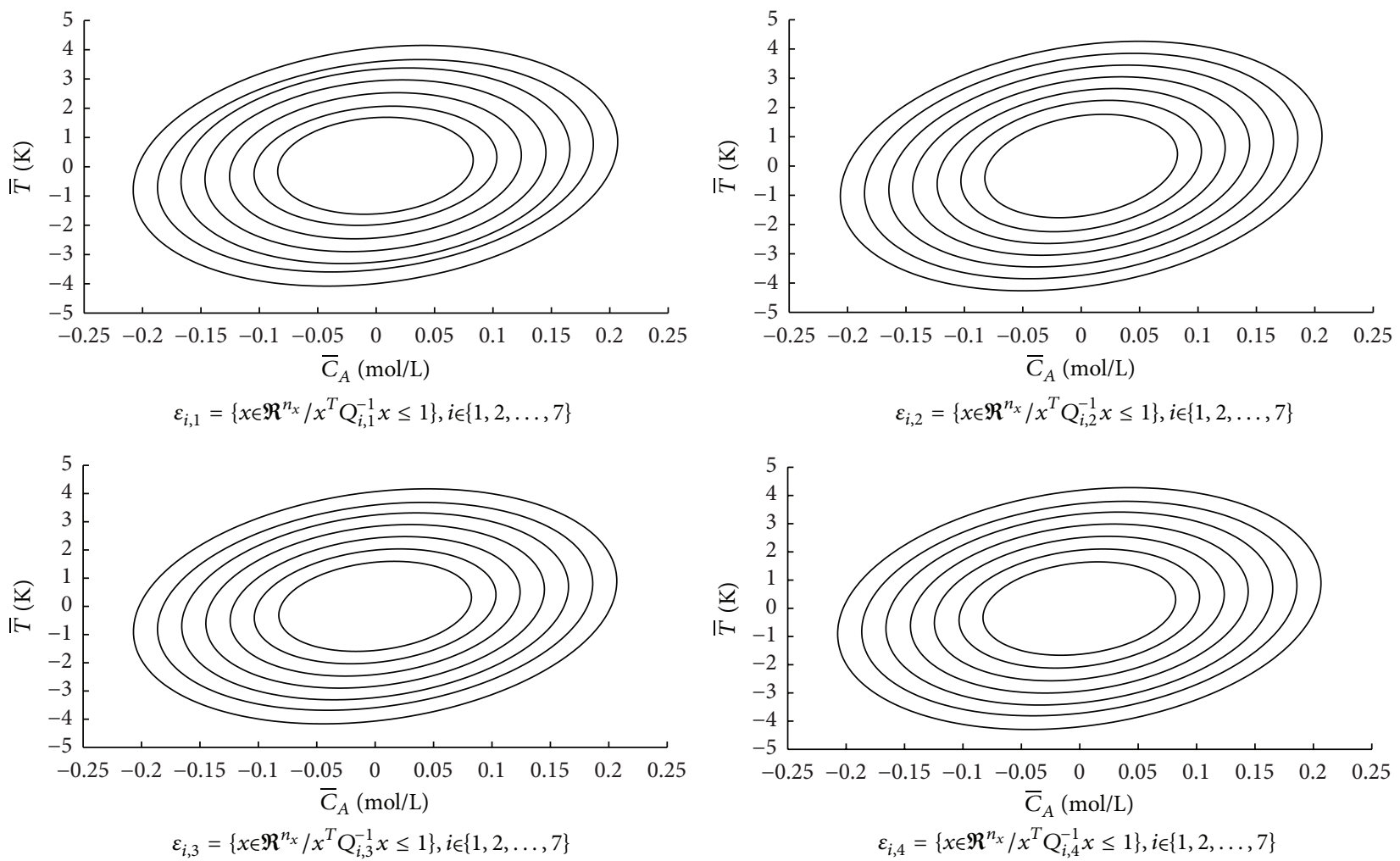

(a) Four sequences of ellipsoids corresponding to $A_{j}$

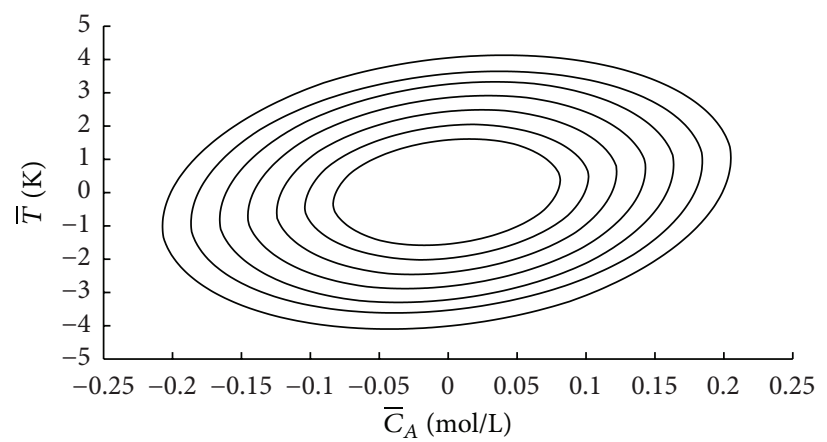

(b) A sequence of positively invariant sets

FIgURE 1: The ellipsoids computed offline in Example 1 (a) four sequences of ellipsoids corresponding to $A_{j}$ (b) a sequence of positively invariant sets.

motor, and $v(k)$ is the disturbance acting on the system. The scheduling parameter $\Delta(k)$ is measurable at each sampling time and its value is varied between 0.1 and 10 . We have that all the solutions of (38) are also the solutions of the following differential inclusion:

$$
\begin{aligned}
{\left[\begin{array}{l}
x_{1}(k+1) \\
x_{2}(k+1)
\end{array}\right] \in } & \left(\sum_{j=1}^{2} p_{j} A_{j}\right)\left[\begin{array}{l}
x_{1}(k) \\
x_{2}(k)
\end{array}\right] \\
& +\left[\begin{array}{c}
0 \\
0.0787
\end{array}\right] u(k)+\left[\begin{array}{l}
0.15 \\
0.15
\end{array}\right] v(k),
\end{aligned}
$$

where $A_{j}$ are given by $A_{1}=\left[\begin{array}{ll}1 & 0.1 \\ 0 & 0.99\end{array}\right], A_{2}=\left[\begin{array}{cc}1 & 0.1 \\ 0 & 0\end{array}\right]$ and $p_{j}$ are given by $p_{1}=(10-\Delta(k)) / 0.9, p_{2}=(\Delta(k)-0.1) / 0.9$.
The objective is to regulate $x_{1}(k)$ and $x_{2}(k)$ by manipulating $u(k)$. The input constraint is $|u(k)| \leq 2$ volts. The symmetric weighting matrices in (10) are $\Psi=\left[\begin{array}{ll}1 & 0 \\ 0 & 0\end{array}\right]$ and $\sigma=0.00002$. The value of $\theta$ in (18) has been fixed at 0.98 which has negligible influence on the control performance. A sampling time is $0.1 \mathrm{~s}$.

Figure 3(a) shows two sequences of ellipsoids corresponding to $A_{j}$. A sequence of positively invariant sets computed by intersection between two sequences of ellipsoids is shown in Figure 3(b).

Figure 4 shows 100 state trajectories of the closed-loop system when $v(k)$ and $\Delta(k)$ are arbitrarily time-varying in the ranges of $-0.01 \leq v(k) \leq 0.01$ and $0.1 \leq \Delta(k) \leq$ 10 . Two initial points $(0.9,0)$ and $(-0.8,0.8)$ are chosen. It 

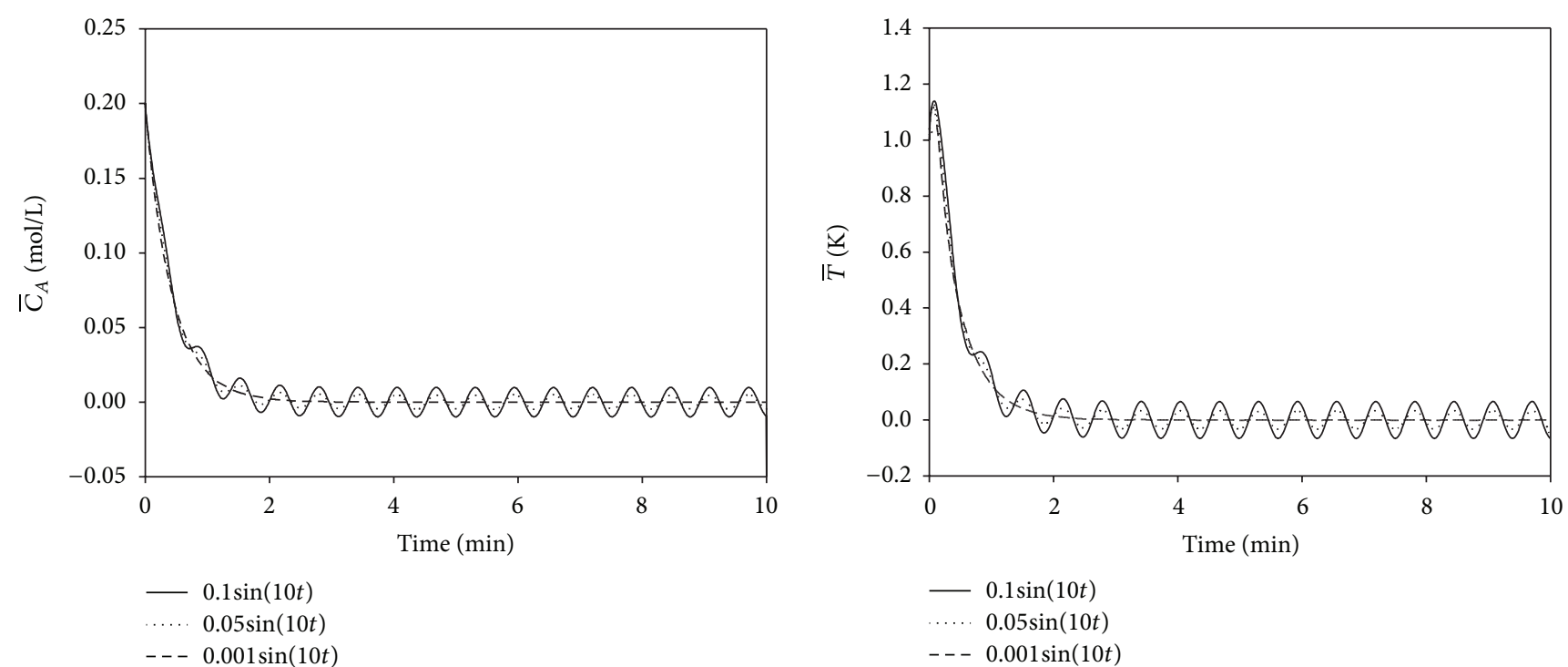

(a) Regulated output

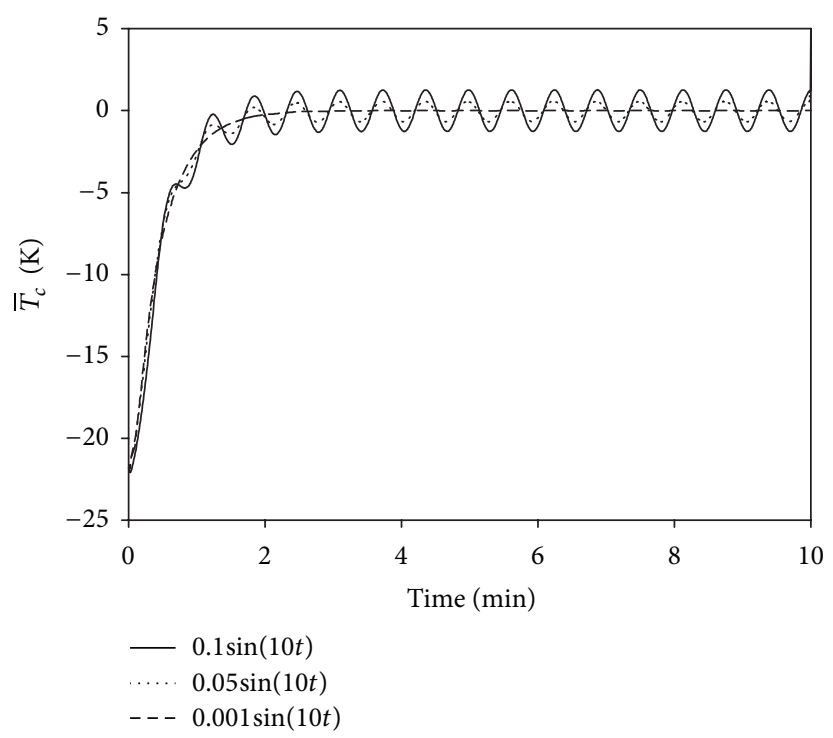

(b) Control input

FIGURE 2: The closed-loop responses in Example 1 (a) regulated output and (b) control input.

can be observed from the figure that all state trajectories are restricted to lie in a sequence of positively invariant sets. The average time for Algorithm 6 to compute a real-time control law is $0.001 \mathrm{~s}$.

\section{Conclusions}

In this paper, we have presented an offline MPC algorithm for constrained LPV systems. The main contribution is to develop an offline MPC algorithm for LPV systems that can deal with both persistent disturbance and time-varying scheduling parameter. The norm-bounding technique is used to derive an offline MPC algorithm based on the parameterdependent state feedback control law and the parameterdependent Lyapunov functions. Most of the optimization problems are solved offline so the algorithm is applicable to fast systems. At each sampling time, a parameter-dependent state feedback control law is calculated by linear interpolation between the precomputed state feedback control laws. The controller design is illustrated with two examples.

\section{Appendices}

\section{A. Proof of Proposition 3}

Lemma A.1 will be used in the proof.

Lemma A.1 (see [23]). Suppose $M>0$ is a symmetric matrix, while $a$ and $b$ are vectors with appropriate dimensions. Then, 

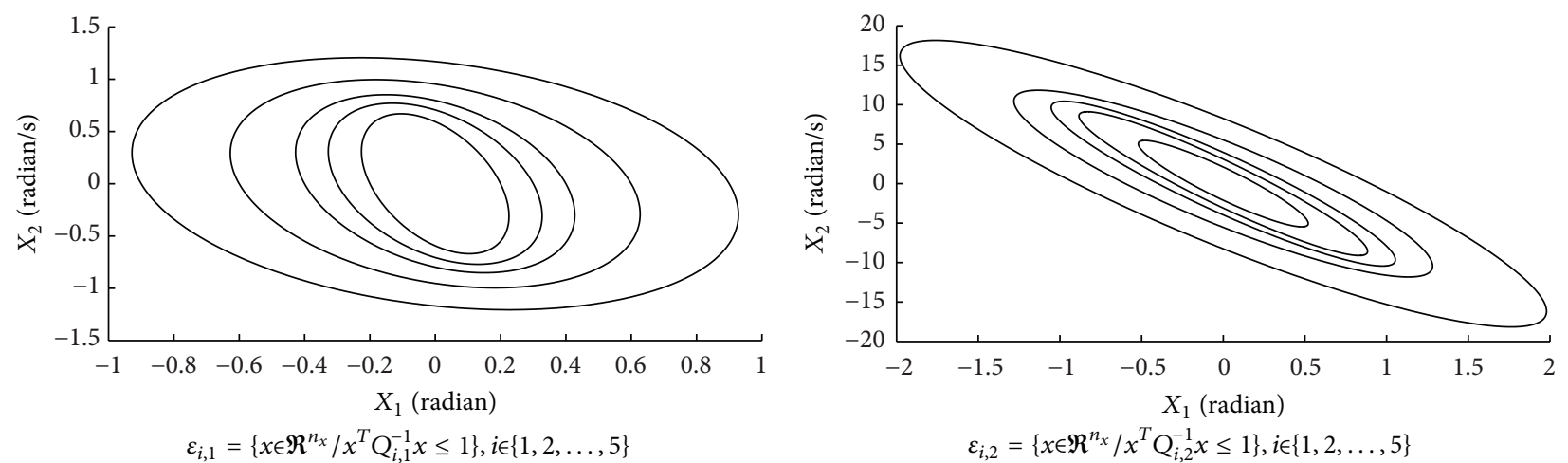

(a) Two sequences of ellipsoids corresponding to $A_{j}$

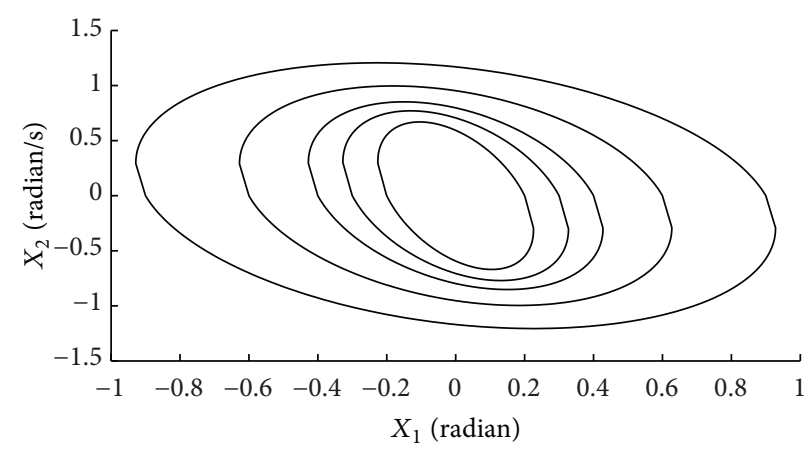

(b) A sequence of positively invariant sets

Figure 3: The ellipsoids computed offline in Example 2 (a) two sequences of ellipsoids corresponding to $A_{j}$ and (b) a sequence of positively invariant sets.

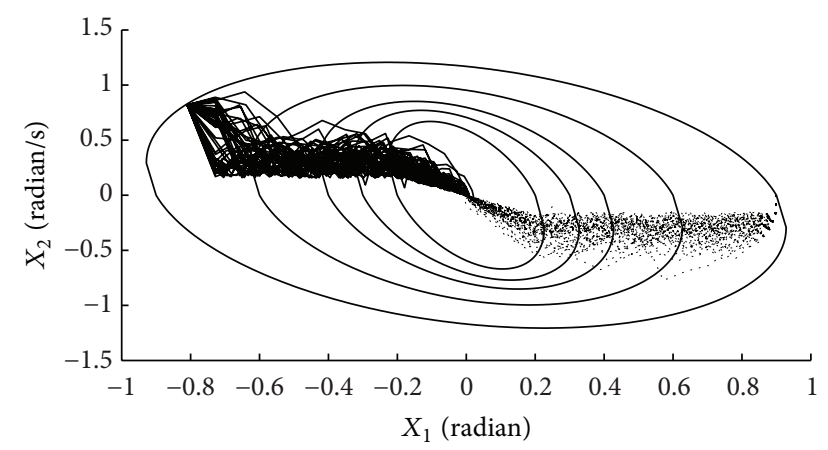

Figure 4: The state trajectories of the closed-loop system in Example 2.

for any scalar $\delta>0,(a+b)^{T} M(a+b) \leq(1+\delta) a^{T} M a+(1+$ $(1 / \delta)) b^{T} M b$.

The norm-bounding technique [23] is used to derive an offline MPC algorithm based on the parameter-dependent state feedback control law and the parameter-dependent Lyapunov functions.

By substituting $x(k+i+1 \mid k)=[A(p(k+i))+B K(p(k+$ $i))] x(k+i \mid k)+D(\alpha(k+i)) v(k+i)$ into (A.1)

$$
\|x(k+i+1 \mid k)\|_{P(p(k+i+1))}^{2} \leq \gamma
$$

TABLE 1: The operating parameters of nonlinear CSTR in Example 1.

\begin{tabular}{lcc}
\hline Parameter & Value & $\mathrm{Unit}$ \\
\hline$q$ & 100 & $\mathrm{~L} / \mathrm{min}$ \\
$T_{f}$ & 350 & $\mathrm{~K}$ \\
$C_{\mathrm{AF}}$ & 1 & $\mathrm{~mol} / \mathrm{L}$ \\
$V$ & 100 & $\mathrm{~L}$ \\
$\rho$ & 1,000 & $\mathrm{~g} / \mathrm{L}$ \\
$C_{p}$ & 0.239 & $\mathrm{~J} /(\mathrm{g} \mathrm{K})$ \\
$\Delta H$ & $-5 \times 10^{4}$ & $\mathrm{~J} / \mathrm{mol}$ \\
$E_{a} / R$ & 8,750 & $\mathrm{~K}$ \\
$k_{o}$ & $7.2 \times 10^{10}$ & $\mathrm{~min}$ \\
$\mathrm{UA}^{-1}$ & $5 \times 10^{4}$ & $\mathrm{~J} /(\mathrm{min} \mathrm{K}$ \\
$C_{A, \mathrm{eq}}$ & 0.5 & $\mathrm{~mol} / \mathrm{L}$ \\
$T_{\text {eq }}$ & 350 & $\mathrm{~K}$ \\
$T_{c, \text { eq }}$ & 300 & $\mathrm{~K}$ \\
$D_{\mathrm{C}}$ & 0.01 & $\mathrm{~mol} /(\mathrm{L} \mathrm{min})$ \\
$D_{T}$ & 0.1 & $\mathrm{~K} / \mathrm{min}$ \\
\hline
\end{tabular}

TABLE 2: The numerical burdens in Example 1.

\begin{tabular}{lcc}
\hline \multirow{2}{*}{ Algorithm } & \multicolumn{2}{c}{ CPU time (s) } \\
& Offline & Online (per step) \\
\hline Algorithm 6 & 20.043 & 0.001 \\
\hline
\end{tabular}


and applying Lemma A.1, for any $\delta_{1}>0$, we see that (A.1) is satisfied if

$$
\begin{aligned}
\left(1+\delta_{1}\right) & \\
& \times\|[A(p(k+i))+B K(p(k+i))] x(k+i \mid k)\|_{P(p(k+i+1)) / \gamma}^{2} \\
& +\left(1+\frac{1}{\delta_{1}}\right)\|D(\alpha(k+i)) v(k+i)\|_{P(p(k+i+1)) / \gamma}^{2} \leq 1 .
\end{aligned}
$$

Consider the term $\|[A(p(k+i))+B K(p(k+i))]$ $x(k+i \mid k) \|_{P(p(k+i+1)) / \gamma}^{2}$ in (A.2); let $\theta\|x(k+i \mid k)\|_{P(p(k+i)) / \gamma}^{2}$ be the maximum value of this term, where $0<\theta<1$ is a prespecified scalar:

$$
\begin{aligned}
& \|[A(p(k+i))+B K(p(k+i))] x(k+i \mid k)\|_{P(p(k+i+1)) / \gamma}^{2} \\
& \quad \leq \theta\|x(k+i \mid k)\|_{P(p(k+i)) / \gamma^{\prime}}^{2}
\end{aligned}
$$

Applying the Schur complement leads to

$$
\left[\begin{array}{cc}
\theta \frac{P(p(k+i))}{\gamma} & * \\
\frac{P(p(k+i+1))}{\gamma}[A(p(k+i))+B K(p(k+i))] & \frac{P(p(k+i+1))}{\gamma}
\end{array}\right] \geq 0 .
$$

From the convexity of the polytopic description, with $P_{j}=$ $\gamma Q_{j}^{-1}, P_{l}=\gamma Q_{l}^{-1}$ and $K_{j}=Y_{j} G_{j}^{-1},($ A.4) is satisfied if

$$
\left[\begin{array}{cc}
\theta Q_{j}^{-1} & * \\
Q_{l}^{-1}\left[A_{j}+B Y_{j} G_{j}^{-1}\right] & Q_{l}^{-1}
\end{array}\right] \geq 0, \quad j, l \in\{1,2, \ldots, L\} .
$$

Multiplying (A.5) from the left by $\operatorname{diag}\left\{G_{j}^{T}, Q_{l}^{T}\right\}$ and from the right by $\operatorname{diag}\left\{G_{j}, Q_{l}\right\}$ and applying $G_{j}^{T} Q_{j}^{-1} G_{j} \geq G_{j}+G_{j}^{T}-Q_{j}$, we obtain LMIs (A.6)

$$
\left[\begin{array}{cc}
\theta\left(G_{j}+G_{j}^{T}-Q_{j}\right) & * \\
A_{j} G_{j}+B Y_{j} & Q_{l}
\end{array}\right] \geq 0, \quad j, l \in\{1,2, \ldots, L\} .
$$

Consider the term $\|D(\alpha(k+i)) v(k+i)\|_{P(p(k+i+1)) / \gamma}^{2}$ in (A.2); let $\xi$ be the maximum value of this term:

$$
\|D(\alpha(k+i)) v(k+i)\|_{P(p(k+i+1)) / \gamma}^{2} \leq \xi .
$$

Applying the Schur complement leads to

$$
\begin{aligned}
& {\left[\frac{P(p(k+i+1))}{\gamma} D(\alpha(k+i)) v(k+i)\right.} \\
& \left.\quad \frac{P(p(k+i+1))}{\gamma}\right] \\
& \quad \geq 0 .
\end{aligned}
$$

From the convexity of the polytopic description, with $P_{l}=$ $\gamma Q_{l}^{-1},($ A.8) is satisfied if

$$
\begin{array}{r}
{\left[\begin{array}{cc}
\xi & * \\
Q_{l}^{-1} D_{t} v_{s} & Q_{l}^{-1}
\end{array}\right] \geq 0, \quad l \in\{1,2, \ldots, L\}, \quad t \in\left\{1,2, \ldots, n_{D}\right\},} \\
s \in\left\{1,2, \ldots, m_{v}\right\} .
\end{array}
$$

Multiplying (A.9) from the left by $\operatorname{diag}\left\{I, Q_{l}\right\}$ and from the right by $\operatorname{diag}\left\{I, Q_{l}\right\}$, we obtain LMIs (A.10):

$$
\left[\begin{array}{cc}
\xi & * \\
D_{t} v_{s} & Q_{l}
\end{array}\right] \geq 0, \quad t \in\left\{1,2, \ldots, n_{D}\right\}, s \in\left\{1,2, \ldots, m_{v}\right\}
$$

$$
l \in\{1,2, \ldots, L\} .
$$

From (A.3) and (A.7), with $\|x(k+i \mid k)\|_{P(p(k+i)) / \gamma}^{2} \leq 1$, (A.2) is equivalent to

$$
\left(1+\delta_{1}\right) \theta+\left(1+\frac{1}{\delta_{1}}\right) \xi \leq 1
$$

The maximum allowable value of $\xi$ can be calculated by solving

$$
\xi=\max _{\delta_{1}} \frac{1-\left(1+\delta_{1}\right) \theta}{\left(1+\left(1 / \delta_{1}\right)\right)}
$$

From (A.12), $\xi$ is obtained as

$$
\xi=\left(1-\theta^{1 / 2}\right)^{2}
$$

\section{B. Proof of Proposition 5 (Input Constraint)}

Defining $\zeta_{h}$ as the $h$ th row of the $n_{u}$-dimensional identity matrix, we have

$$
\max _{i \geq 0}\left|\zeta_{h} u(k+i \mid k)\right|^{2}=\max _{i \geq 0}\left|\zeta_{h} K(p(k+i)) x(k+i \mid k)\right|^{2}
$$


Applying Cauchy-Schwarz inequality, with ||$x(k+i \mid$ $k) \|_{P(p(k+i)) / \gamma}^{2} \leq 1$, leads to

$$
\begin{aligned}
& \max _{i \geq 0}\left|\zeta_{h} K(p(k+i)) x(k+i \mid k)\right|^{2} \\
& \leq\left\|\zeta_{h} K(p(k+i))\left(\frac{P(p(k+i))}{\gamma}\right)^{-1 / 2}\right\|^{2} .
\end{aligned}
$$

The input constraint is satisfied if

$$
\begin{array}{r}
\left\|\zeta_{h} K(p(k+i))\left(\frac{P(p(k+i))}{\gamma}\right)^{-1 / 2}\right\|^{2} \leq \bar{u}_{h}^{2}, \\
h \in\left\{1,2, \ldots, n_{u}\right\} .
\end{array}
$$

By applying the Schur complement, (B.3) is equivalent to

$$
\begin{gathered}
{\left[\begin{array}{c}
\chi \\
K(p(k+i))^{T}
\end{array}\left(\frac{P(p(k+i))}{\gamma}\right)\right] \geq 0,} \\
\chi_{h h} \leq \bar{u}_{h}^{2}, \quad h \in\left\{1,2, \ldots, n_{u}\right\} .
\end{gathered}
$$

From the convexity of the polytopic description, with $P_{j}=$ $\gamma Q_{j}^{-1}$ and $K_{j}=Y_{j} G_{j}^{-1},($ B.4 $)$ is satisfied if

$$
\begin{aligned}
& {\left[\begin{array}{cc}
\chi & * \\
\left(Y_{j} G_{j}^{-1}\right)^{T} & Q_{j}^{-1}
\end{array}\right] \geq 0, \quad \chi_{h h} \leq \bar{u}_{h}^{2},} \\
& h \in\left\{1,2, \ldots, n_{u}\right\}, \quad j \in\{1,2, \ldots, L\} .
\end{aligned}
$$

Premultiplying by $\operatorname{diag}\left\{I, G_{j}^{T}\right\}$, postmultiplying by $\operatorname{diag}\left\{I, G_{j}\right\}$ and applying $G_{j}^{T} Q_{j}^{-1} G_{j} \geq G_{j}+G_{j}^{T}-Q_{j}$, we obtain LMIs (B.6)

$$
\begin{gathered}
{\left[\begin{array}{cc}
\chi & * \\
Y_{j}^{T} & G_{j}+G_{j}^{T}-Q_{j}
\end{array}\right] \geq 0, \quad \chi_{h h} \leq \bar{u}_{h}^{2},} \\
h \in\left\{1,2, \ldots, n_{u}\right\}, \quad j \in\{1,2, \ldots, L\} .
\end{gathered}
$$

Since $\|x(k+i \mid k)\|_{P(p(k+i)) / \gamma}^{2} \leq 1$, applying the Schur complement leads to

Substituting $x(k+i+1 \mid k)=[A(p(k+i))+B K(p(k+$ $i))] x(k+i \mid k)+D(\alpha(k+i)) v(k+i)$ and applying CauchySchwarz inequality and Lemma A.1, for any $\delta_{2}>0$, we see that the output constraint is satisfied if

$$
\begin{aligned}
&\left(1+\delta_{2}\right) \|_{r} C(p(k+i+1)) \\
& \times[A(p(k+i))+B K(p(k+i))] x(k+i \mid k) \|^{2} \\
&+\left(1+\frac{1}{\delta_{2}}\right)\left\|\zeta_{r} C(p(k+i+1)) D(\alpha(k+i)) v(k+i)\right\|^{2} \leq \bar{y}_{r}^{2} .
\end{aligned}
$$

Consider the term $\| \zeta_{r} C(p(k+i+1))[A(p(k+i))+B K(p(k+$ $i))] x(k+i \mid k) \|^{2}$ in (C.2); let $\Xi_{r}$ be the maximum value of this term

$$
\begin{aligned}
& \| \zeta_{r} C(p(k+i+1))[A(p(k+i))+B K(p(k+i))] \\
& \quad \times x(k+i \mid k) \|^{2} \leq \Xi_{r} .
\end{aligned}
$$

$$
\begin{aligned}
\max _{i \geq 0}\left|\zeta_{r} y(k+i+1 \mid k)\right|^{2} & \\
& =\max _{i \geq 0}\left|\zeta_{r} C(p(k+i+1)) x(k+i+1 \mid k)\right|^{2} .
\end{aligned}
$$

Defining $\zeta_{r}$ as the $r$ th row of the $n_{y}$-dimensional identity matrix, we have

$$
\begin{gathered}
{\left[[A(p(k+i))+B K(p(k+i))]^{T} C(p(k+i+1))^{T}\left(\frac{P(p(k+i))}{\gamma}\right)\right] \geq 0} \\
\Gamma_{r r} \leq \Xi_{r}, \quad r \in\left\{1,2, \ldots, n_{y}\right\} .
\end{gathered}
$$

From the convexity of the polytopic description, with $P_{j}=$ $\gamma Q_{j}^{-1}$ and $K_{j}=Y_{j} G_{j}^{-1},(\mathrm{C} .4)$ is satisfied if

$$
\begin{gathered}
{\left[\begin{array}{cc}
\Gamma & * \\
{\left[A_{j}+B Y_{j} G_{j}^{-1}\right]^{T} C_{l}^{T}} & Q_{j}^{-1}
\end{array}\right] \geq 0, \quad \Gamma_{r r} \leq \Xi_{r},} \\
r \in\left\{1,2, \ldots, n_{y}\right\}, \quad j, l \in\{1,2, \ldots, L\}
\end{gathered}
$$

Premultiplying by $\operatorname{diag}\left\{I, G_{j}^{T}\right\}$, postmultiplying by $\operatorname{diag}\{I$, $G_{j}$, and applying $G_{j}^{T} Q_{j}^{-1} G_{j} \geq G_{j}+G_{j}^{T}-Q_{j}$, we obtain LMIs (C.6)

$$
\begin{gathered}
{\left[\begin{array}{c}
\Gamma \quad * \\
\left(A_{j} G_{j}+B Y_{j}\right)^{T} C_{l}^{T} \quad G_{j}+G_{j}^{T}-Q_{j}
\end{array}\right] \geq 0, \quad \Gamma_{r r} \leq \Xi_{r},} \\
r \in\left\{1,2, \ldots, n_{y}\right\}, \quad j, l \in\{1,2, \ldots, L\} .
\end{gathered}
$$


Consider the term $\left\|\zeta_{r} C(p(k+i+1)) D(\alpha(k+i)) v(k+i)\right\|^{2}$ in (C.2); let $\Phi_{r}$ be the maximum value of this term:

$$
\left\|\zeta_{r} C(p(k+i+1)) D(\alpha(k+i)) v(k+i)\right\|^{2} \leq \Phi_{r} .
$$

By applying the Schur complement, we obtain

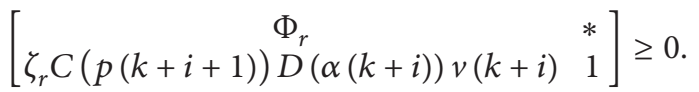

From the convexity of the polytopic description, (C.8) is satisfied if

$$
\begin{gathered}
{\left[\begin{array}{cc}
\Phi_{r} & * \\
C_{r, l} D_{t} v_{s} & 1
\end{array}\right] \geq 0, \quad r \in\left\{1,2, \ldots, n_{y}\right\}, l \in\{1,2, \ldots, L\}} \\
t \in\left\{1,2, \ldots, n_{D}\right\}, \quad s \in\left\{1,2, \ldots, m_{v}\right\}
\end{gathered}
$$

where $C_{r, l}$ is the $r$ th row of $C_{l}$. Thus, $\Phi_{r}$ can be calculated by solving

$$
\min _{\Phi_{r}} \Phi_{r} \quad \text { s.t. (C.9) }
$$

From (C.3) and (C.7), (C.2) is equivalent to

$$
\left(1+\delta_{2}\right) \Xi_{r}+\left(1+\frac{1}{\delta_{2}}\right) \Phi_{r} \leq \bar{y}_{r}^{2} .
$$

The maximum allowable value of $\Xi_{r}$ can be calculated by solving

$$
\Xi_{r}=\max _{\delta_{2}} \frac{\bar{y}_{r}^{2}-\left(1+\left(1 / \delta_{2}\right)\right) \Phi_{r}}{\left(1+\delta_{2}\right)} .
$$

From (C.12), $\Xi_{r}$ is obtained as

$$
\Xi_{r}=\left(\bar{y}_{r}-\Phi_{r}^{1 / 2}\right)^{2}
$$

\section{Conflict of Interests}

The author declares that there is no conflict of interests regarding the publication of this paper.

\section{Acknowledgment}

This research project is supported by Mahidol University.

\section{References}

[1] M. Morari and J. H. Lee, "Model predictive control: past, present and future," Computers and Chemical Engineering, vol. 23, no. 45, pp. 667-682, 1999.

[2] D. Q. Mayne, J. B. Rawlings, C. V. Rao, and P. O. M. Scokaert, "Constrained model predictive control: stability and optimality," Automatica, vol. 36, no. 6, pp. 789-814, 2000.

[3] S. J. Qin and T. A. Badgwell, "A survey of industrial model predictive control technology," Control Engineering Practice, vol. 11, no. 7, pp. 733-764, 2003.
[4] M. V. Kothare, V. Balakrishnan, and M. Morari, "Robust constrained model predictive control using linear matrix inequalities," Automatica, vol. 32, no. 10, pp. 1361-1379, 1996.

[5] E. F. Camacho and C. Bordons, Model Predictive Control, Springer, London, UK, 2nd edition, 2004.

[6] M. Jungers, R. C. L. F. Oliveira, and P. L. D. Peres, "MPC and LPV systems with bounded parameter variations," International Journal of Control, vol. 84, no. 1, pp. 24-36, 2011.

[7] S. Yu, C. Böhm, H. Chen, and F. Allgöwer, "Model predictive control of constrained LPV systems," International Journal of Control, vol. 85, no. 6, pp. 671-683, 2012.

[8] S. Boyd and L. Vandenberghe, Convex Optimization, Cambridge University Press, Cambridge, UK, 1st edition, 2004.

[9] Y. Lu and Y. Arkun, "Quasi-min-max MPC algorithms for LPV systems," Automatica, vol. 36, no. 4, pp. 527-540, 2000.

[10] N. Wada, K. Saito, and M. Saeki, "Model predictive control for linear parameter varying systems using parameter dependent Lyapunov function," IEEE Transactions on Circuits and Systems II: Express Briefs, vol. 53, no. 12, pp. 1446-1450, 2006.

[11] A. Casavola, D. Famularo, and G. Franzè, "A feedback min-max MPC algorithm for LPV systems subject to bounded rates of change of parameters," IEEE Transactions on Automatic Control, vol. 47, no. 7, pp. 1147-1153, 2002.

[12] P. Park and S. C. Jeong, "Constrained RHC for LPV systems with bounded rates of parameter variations," Automatica, vol. 40, no. 5, pp. 865-872, 2004.

[13] H. Suzuki and T. Sugie, "Model predictive control for linear parameter varying constrained systems using ellipsoidal set prediction," International Journal of Control, vol. 80, no. 2, pp. 314-321, 2007.

[14] A. C. Brooms, B. Kouvaritakis, and Y. I. Lee, "Constrained MPC for uncertain linear systems with ellipsoidal target sets," Systems \& Control Letters, vol. 44, no. 3, pp. 157-166, 2001.

[15] P. Bumroongsri and S. Kheawhom, "An ellipsoidal off-line model predictive control strategy for linear parameter varying systems with applications in chemical processes," Systems \& Control Letters, vol. 61, no. 3, pp. 435-442, 2012.

[16] T. Besselmann, J. Löfberg, and M. Morari, "Explicit MPC for LPV systems: stability and optimality," IEEE Transactions on Automatic Control, vol. 57, no. 9, pp. 2322-2332, 2012.

[17] B. Ding, "Quadratic boundedness via dynamic output feedback for constrained nonlinear systems in Takagi-Sugeno's form," Automatica, vol. 45, no. 9, pp. 2093-2098, 2009.

[18] B. Ding, "Constrained robust model predictive control via parameter-dependent dynamic output feedback," Automatica, vol. 46, no. 9, pp. 1517-1523, 2010.

[19] W. Langson, I. Chryssochoos, S. V. Raković, and D. Q. Mayne, "Robust model predictive control using tubes," Automatica, vol. 40, no. 1, pp. 125-133, 2004.

[20] D. Limon, I. Alvarado, T. Alamo, and E. F. Camacho, "Robust tube-based MPC for tracking of constrained linear systems with additive disturbances," Journal of Process Control, vol. 20, no. 3, pp. 248-260, 2010.

[21] R. Gonzalez, M. Fiacchini, T. Alamo, J. L. Guzman, and F. Rodriguez, "Online robust tube-based MPC for time-varying systems: a practical approach," International Journal of Control, vol. 84, no. 6, pp. 1157-1170, 2011.

[22] Z. Wan and M. V. Kothare, "An efficient off-line formulation of robust model predictive control using linear matrix inequalities," Automatica, vol. 39, no. 5, pp. 837-846, 2003. 
[23] B. C. Ding, Y. Xi, M. T. Cychowski, and T. O’Mahony, "A synthesis approach for output feedback robust constrained model predictive control," Automatica, vol. 44, no. 1, pp. 258264, 2008.

[24] J. F. Sturm, "Using SeDuMi 1.02, a MATLAB toolbox for optimization over symmetric cones," Optimization Methods and Software, vol. 11/12, no. 1-4, pp. 625-653, 1999.

[25] J. Löfberg, "Automatic robust convex programming," Optimization Methods \& Software, vol. 27, no. 1, pp. 115-129, 2012.

[26] B. Ding and B. Huang, "Reformulation of LMI-based stabilisation conditions for non-linear systems in Takagi-Sugeno's form," International Journal of Systems Science. Principles and Applications of Systems and Integration, vol. 39, no. 5, pp. 487496, 2008. 


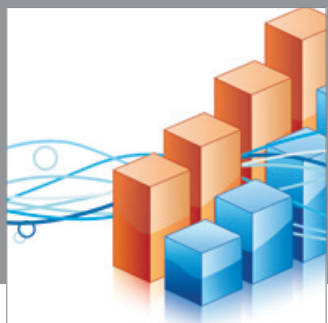

Advances in

Operations Research

mansans

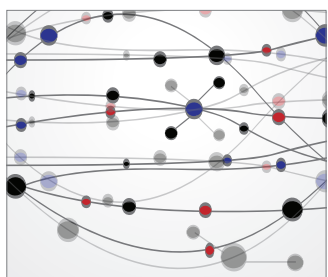

The Scientific World Journal
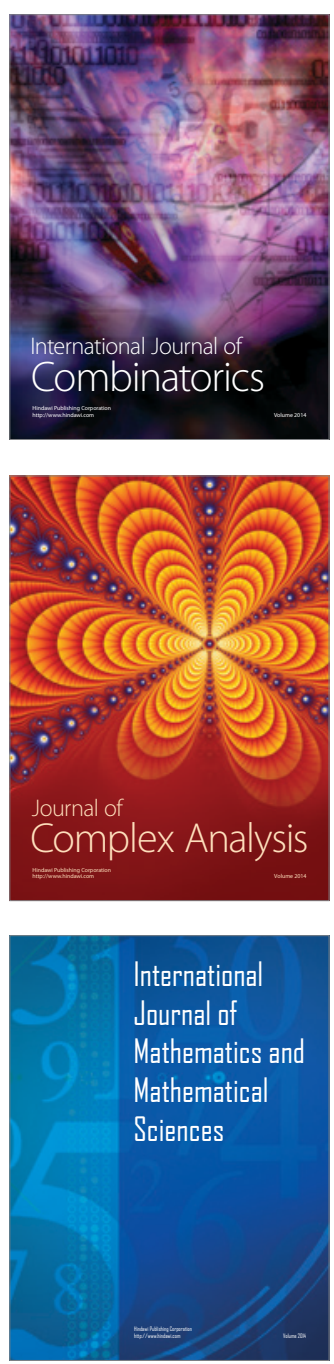
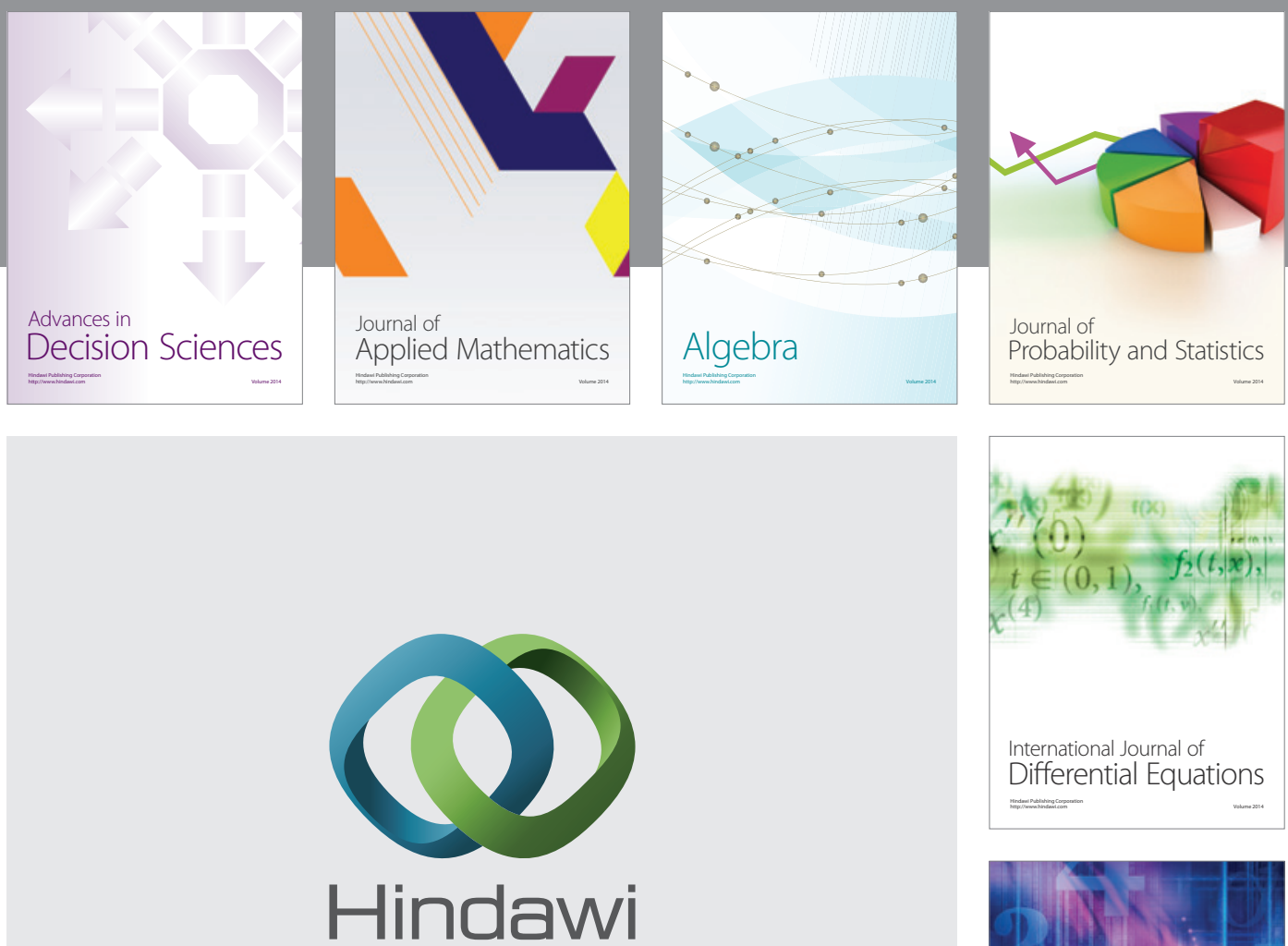

Submit your manuscripts at http://www.hindawi.com
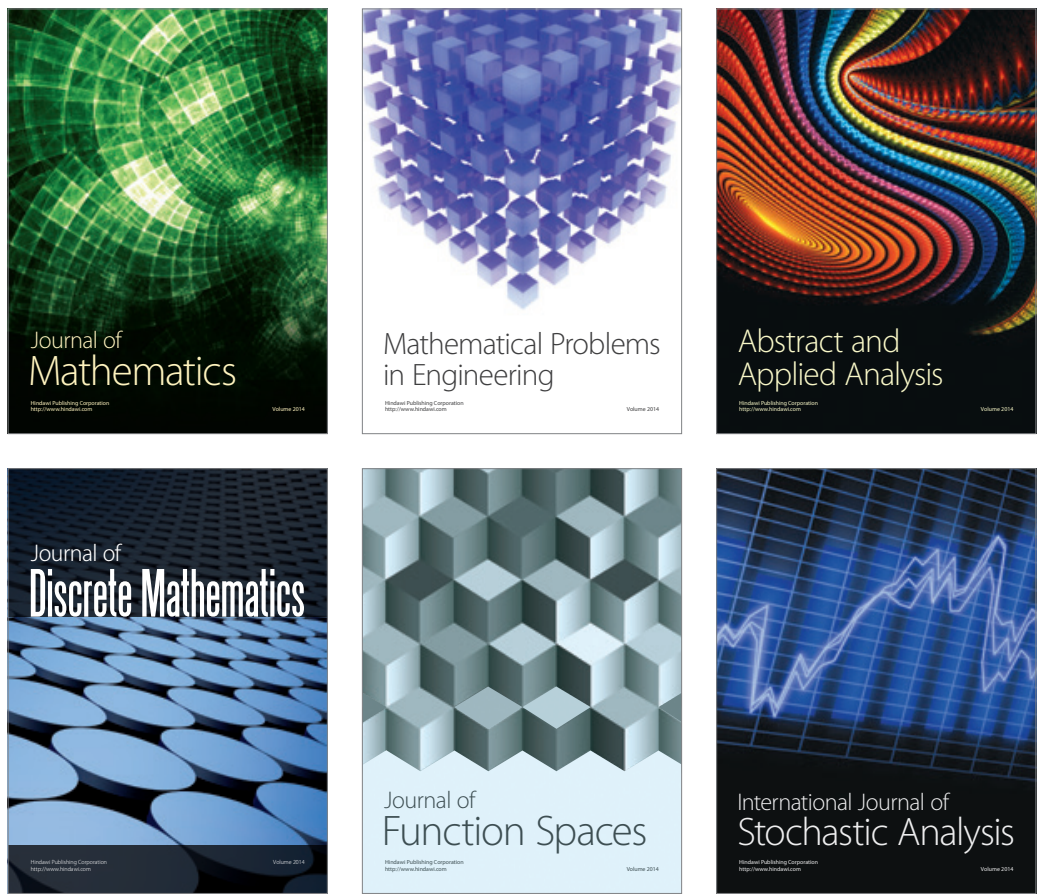

Journal of

Function Spaces

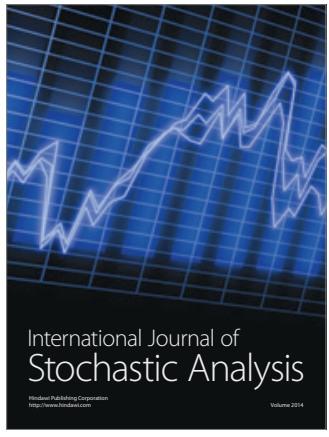

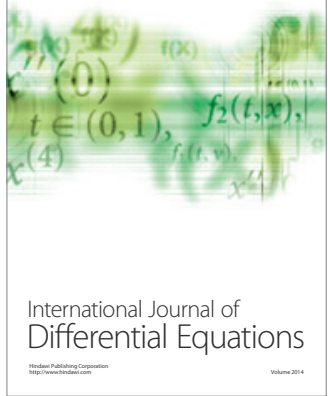
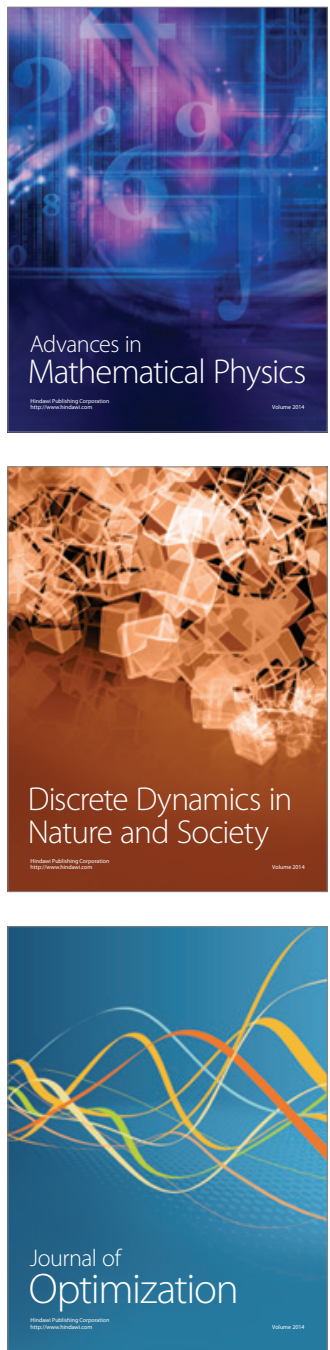